\title{
Fibrin glue: Novel submucosal injection agent for endoscopic submucosal dissection $\square$
}

\section{(C) (i) $\odot$}

\author{
Authors \\ Madoka Takao', Yoshitaka Takegawa², Toshitatsu Takao ${ }^{1}$, Hiroya Sakaguchi ${ }^{1}$, Yoshiko Nakano ${ }^{1}$, Shinwa Tanaka ${ }^{1}$, \\ Yoshinori Morita', Takashi Toyonaga ${ }^{3}$, Eiji Umegaki ${ }^{1}$, Hiromu Kutsumi ${ }^{4}$, Yuzo Kodama ${ }^{1}$
}

Institutions

1 Division of Gastroenterology, Department of Internal Medicine, Kobe University Graduate School of Medicine, Kobe, Hyogo, Japan

2 Research \& Development Division, Medical Affairs Section, KM Biologics Co., Ltd., Kumamoto, Japan

3 Department of Endoscopy, Kobe University Hospital, Kobe, Hyogo, Japan

4 Center for Clinical Research and Advanced Medicine, Shiga University of Medical Science, Shiga, Otsu, Japan

submitted 20.6.2020

accepted after revision $\quad 5.10 .2020$

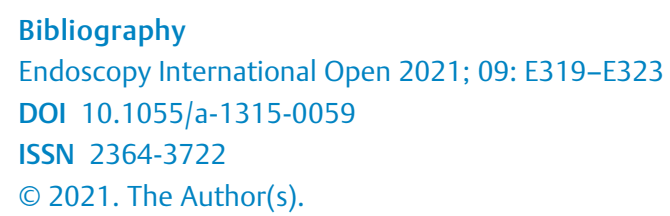

This is an open access article published by Thieme under the terms of the Creative Commons Attribution-NonDerivative-NonCommercial License, permitting copying and reproduction so long as the original work is given appropriate credit. Contents may not be used for commecial purposes, or adapted, remixed, transformed or built upon. (https://creativecommons.org/licenses/by-nc-nd/4.0/)

Georg Thieme Verlag KG, Rüdigerstraße 14,

70469 Stuttgart, Germany

Corresponding author

Toshitatsu Takao, MD, PhD, Division of Gastroenterology, Department of Internal Medicine, Kobe University Graduate School of Medicine, 7-5-1 Kusunokicho, Chuo-ku, Kobe-shi,

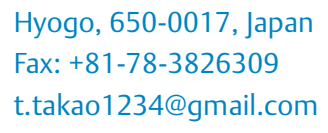

\section{ABSTRACT}

Background and study aims Adequate mucosal elevation by submucosal injection is crucial for patient safety and efficiency during endoscopic submucosal dissection (ESD). This study aimed to evaluate the efficacy of fibrin glue (FG) as a long-lasting submucosal injection agent and to evaluate the technical feasibility of FG injection for ESD.

Materials and methods To compare the capabilities of different agents in maintaining submucosal evaluation, we injected FG, hyaluronic acid solution, and normal saline into the porcine gastric specimen that was incised into approximately $5 \times 5 \mathrm{~cm}$ squares. Then, we measured the height of submucosal elevations over time. Moreover, three hypothetical lesions from the resected porcine stomach underwent ESD with FG injection. Thereafter, we conducted macroscopic and histopathologic analyses.

Results FG maintained the greatest submucosal elevation among all the injection agents. Three ESD procedures were performed with en bloc resection. Both macroscopic and histopathologic findings showed a thick FG clot on the ulcers.

Conclusions The FG solution can be potentially used as an ESD submucosal injection agent in an in vitro model.

\section{Introduction}

Endoscopic submucosal dissection (ESD) is a reliable therapeutic strategy for removing gastrointestinal neoplasms. However, several complications, such as perforation and bleeding, are still reported [1]. Creating adequate and longer-lasting submucosal elevation by submucosal injection of a solution is important to prevent perforation and to make the procedure safer and easier. Submucosal injection of several types of solutions, such as normal saline (NS) and hyaluronic acid (HA), is performed for ESD [2]. However, submucosal fluid leakage from in- jection sites is unavoidable; it decreases submucosal elevation. Hence, a longer-lasting submucosal injection agent might be beneficial.

Fibrin glue (FG) has been used widely as a safe and effective agent in multiple surgical fields for tissue adhesion, suture site reinforcement, and hemostasis [3]. In endoscopy, FG has been used in Europe for approximately 20 years as a safe submucosal injection agent for hemostasis in bleeding ulcer cases [4-6]. FG is a blood-derived product that contains two component solutions: fibrinogen and thrombin solution. When mixed, it creates a stable clot that is not absorbed rapidly into the surrounding 
tissues, contrary to other submucosal injection solutions [7]. Therefore, we hypothesized that FG might be a longer-lasting submucosal injection agent for ESD.

This study, which used a resected porcine stomach, aimed to evaluate the ability of FG to maintain submucosal elevation and to evaluate its technical feasibility when used as an ESD submucosal injection agent.

\section{Material and methods}

\section{Preliminary study: Comparison of submucosal injection agent ability to maintain submucosal elevation}

The ability of FG (Bolheal; KM Biologics Co., Ltd.) to maintain submucosal elevation was compared with those of $\mathrm{HA}$ (MucoUP; Boston Scientific.) and NS. Preparation of the resected porcine gastric specimen and the injection methods were demonstrated according to the study by Fujishiro et al, which evaluated the lesion lifting properties of several submucosal injection agents [2]. Considering that FG contains fibrinogen and thrombin solutions, $1 \mathrm{~mL}$ of each solution ( $2 \mathrm{~mL}$ total) was injected into the submucosal layer. Accordingly, the injection volume of $\mathrm{HA}$ and NS was adjusted to $2 \mathrm{~mL}$. After injecting each solution, we recorded the submucosal elevation immediately after, and 5, 10, 20, 25, 30, 45, and 60 minutes after injections. Then, images were taken from the same distance using a fixed camera (Q7; Pentax Corp.). Using an image analysis software (Hakarundesu v. 0.7.1, onegland.net), we measured the height of each submucosal elevation. The same procedure was conducted five times individually for each submucosal injection solution. Thereafter, we compared the mean submucosal elevation of the three submucosal injection solutions at each time point.

\section{Feasibility study: ESD conducted in ex vivo porcine stomach}

We performed ESD using FG as the submucosal injection agent in the resected porcine stomach. An endoscopist, who had an experience of more than 100 ESD cases, performed the procedures. All procedures were performed using an upper gastrointestinal endoscope (GIF-H260; Olympus Corp.). An electric knife (Flush knife BT DK2618JB; Fujifilm, or IT knife 2, KD-611L; Olympus Corp.) and an electrical generator (ESG-400; Olympus Corp.) were also used. Three ESD procedures were performed for three hypothetical lesions of approximately $20 \mathrm{~mm}$, following the same process. To enhance the visibility, the FG solution was stained with indigo carmine. When starting submucosal injection, a small amount of NS was injected at the first site to ensure that the needle tip was inserted into the submucosal layer to prevent the loss of FG solution. Then, the fibrinogen solution was injected into the submucosal layer, followed by the thrombin solution immediately; different injection needles (25G, 4 mm; Impact flow $\mathrm{H}$ type SB, Top) were used to avoid creating a clot inside the catheter. Then, submucosal dissection was performed in the middle layer of the FG clot. The procedure time from the start to the completion of submucosal dissection and the total amount of FG solution used for ESD in each hypotheti- cal lesion were recorded. Afterward, we examined the ESD ulcer sites macroscopically and histopathologically.

\section{Statistical analysis}

Means, standard deviations and $95 \%$ confidence intervals were calculated using EZR (Saitama Medical Center, Jichi Medical University Site, Saitama, Japan). EZR is a graphical user interface for $R$ (The R Foundation for Statistical Computing, Vienna, Austria, version 2.13.0).

\section{Results}

\section{Preliminary study: Comparison of submucosal injection agents' ability to maintain submucosal elevation}

The results of the chronological changes in height and shape of submucosal elevation for each agent are shown individually in $>$ Fig. 1, > Fig. 2, and $>$ Table 1. We found that use of FG and $\mathrm{HA}$ resulted in similar changes in height of submucosal elevation immediately after the injections. The mean (95\% confidence interval) height immediately after the injection was $6.2 \mathrm{~mm}$ (range, 4.9 to 7.6 ) for FG, $6.3 \mathrm{~mm}$ (range, 5.7 to 7.0 ) for $\mathrm{HA}$, and $5.5 \mathrm{~mm}$ (range, 3.7 to 7.2 ) for NS. Regarding the duration of maintaining submucosal elevation, FG maintained an elevation that was higher than that of the other solutions. The height of submucosal elevation 60 minutes after FG injection was $5.6 \mathrm{~mm}$ (range, 4.7 to 6.5), while that of HA and NS injections considerably decreased to $2.7 \mathrm{~mm}$ (range, 2.5 to 3.0 ) and $1.6 \mathrm{~mm}$ (range, 0.0 to 3.1 ), respectively. FG solution created a stable clot and did not spread into the surrounding tissues, whereas the other solutions spread into the submucosal layer over time (\Fig. 2 ).

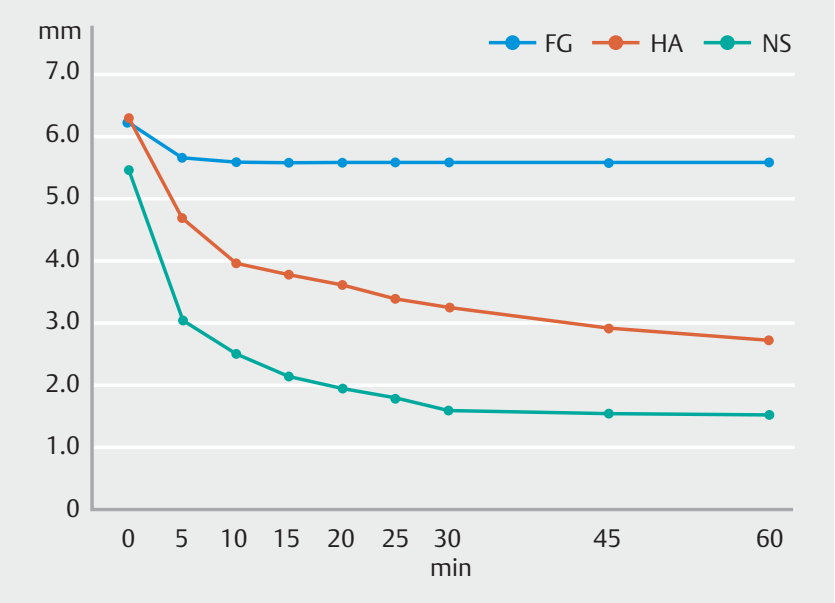

- Fig. 1 Chronological changes in the height of submucosal elevation after the injection of the following submucosal injection agents: FG, fibrin glue $(n=5)$; HA, hyaluronic acid $(n=5)$; and NS, normal saline $(n=5)$. Both FG and HA created a greater submucosal elevation immediately after the injections compared with NS, but FG maintained the greatest submucosal elevation. 

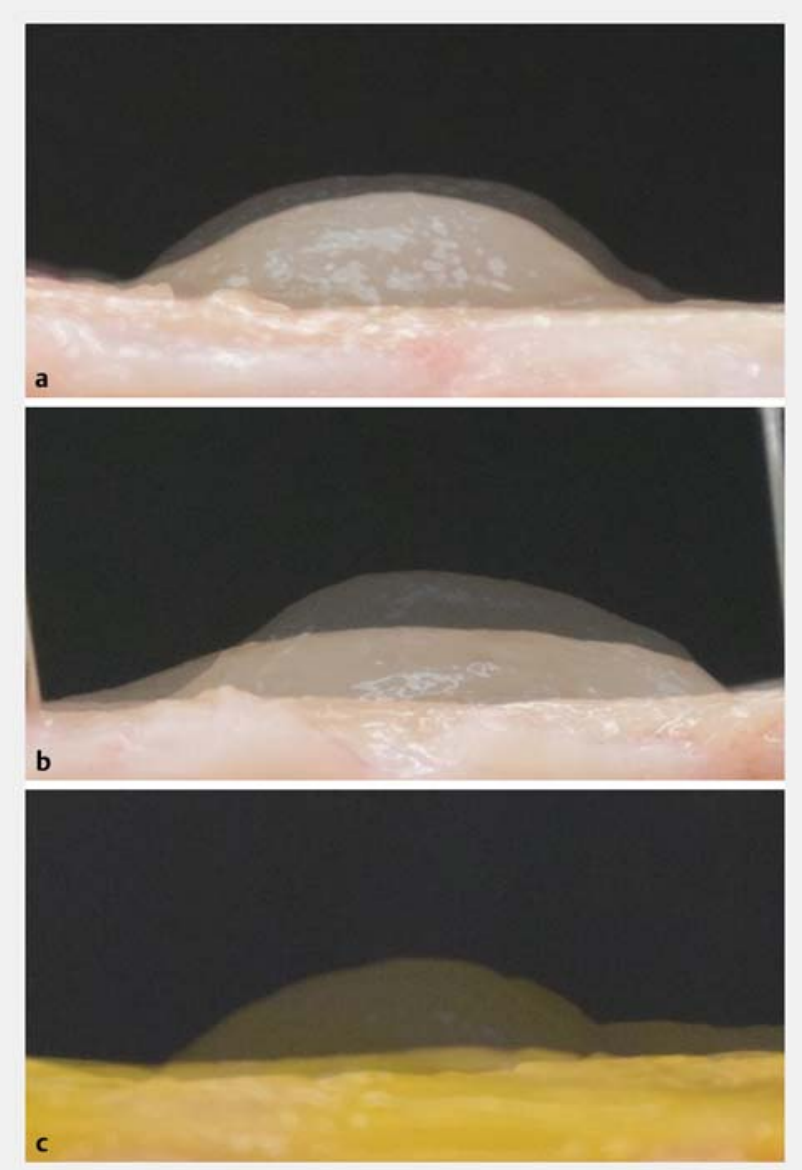

- Fig. 2 Chronological changes in the submucosal elevation for each submucosal injection agent. a Fibrin glue (FG). b Hyaluronic acid (HA). c Normal saline (NS). The image of the mucosa before injection and the images of submucosal elevation immediately after and 60 minutes after injection were superimposed with $50 \%$ transmittance. FG injection maintained the greatest submucosal elevation among the submucosal injection agents.

\section{Feasibility study: ESD conducted in ex vivo porcine stomach}

The three ESD procedures using FG submucosal injection were performed with en bloc resection ( $\bullet$ Video 1 ). The procedure times were 10 minute, 7 seconds; 26 minute, 23 seconds, and 9 minutes, 50 seconds, with total injection volumes of 3.5, 3.6, and $3.8 \mathrm{~mL}$, respectively ( $\bullet$ Table $\mathbf{1}$ ). Additional submucosal injections were not necessary during the ESD procedures. No thermal damage or incision to the mucosa was found on the resected specimen ( $\bullet \mathbf{F i g} \cdot \mathbf{3 b}$ ). Both macroscopic and histopathologic findings revealed thick FG clots that were created on the ulcer sites ( $\vee$ Fig. 3 a and $\triangleright$ Fig. $\mathbf{3 b}$ ).

\section{Discussion}

This study is the first to examine the feasibility of FG solution used as a submucosal injection agent for ESD. We found that the FG submucosal injection could create adequate submucosal elevation that was superior to any other tested injection agents used in maintaining submucosal elevation. Although the height of the submucosal elevation created by FG decreased slightly at 5 minutes after the initial injection, it then maintained its height until approximately 60 minutes later.

Meanwhile, the ESD procedures using FG submucosal injections were completed without difficulty. In addition, adequate elevation was maintained by the FG clots in the submucosal layer during the procedures. Hence, FG might contribute to performing easier and safer ESD, shortening procedure times by reducing the time necessary to perform additional injections and reducing the risks of bleeding caused by damage to blood vessels in the submucosal layer with the injection needle. Meanwhile, one of the gastric lesions (lesion no. 2) required a longer procedure time than the other lesions ( $>$ Table 2 ) because of the rigidity of the FG clot. The FG clot became rigid in some parts, which were hard to dissect smoothly. The density of FG clot depends on the fibrinogen concentration. In this study, the fibrinogen and thrombin solutions were injected separately, which may have caused uneven distribution of fibrinogen concentration within the FG clot. For example, the development of a double-lumen local injection needle may solve this problem by allowing for more uniform injections.

Additional submucosal injections were not necessary in the present study as the lesions were small. However, additional injections are required for larger lesions. Moreover, it is important to evaluate the availability of additional injections for cases with fibrosis. Further investigations are necessary to examine how to administer injections effectively as well as strategies to administer injections to larger lesions and lesions with fibrosis. Regarding devices, non-insulated knives or forceps-like devices could be suitable because, in the present study, it was difficult to insert an insulated device into the rigid part of the FG clots. Developing a new resection device suitable for the FG solution might be desirable to perform the procedure more effectively.

Moreover, both macroscopic and histopathologic findings indicated that the ESD ulcers were covered with a thick FG clot, which indicates the possibility of the covering effects of the clot. The clot might prevent intraoperative and postoperative bleeding as well as delayed perforation by preventing exposure to the digestive juices. Endoscopic covering using FG and polyglycolic acid sheet has attracted attention as a method of preventing ESD-related adverse events [8,9]. Currently applied covering methods using FG and polyglycolic acid require high endoscopic skills and the procedure time is relatively long [10]. Results show that ESD using FG submucosal injection might overcome these issues because this method is technically easy. In addition, when used as a submucosal injection agent for hemostasis in patients with bleeding ulcers, the FG clot is regarded as a long-lasting vessel compressant that minimizes the bleeding [7]. Therefore, the FG clot itself may possess hemostatic functionality. Moreover, there is a possibility that the FG submucosal injections might prevent the muscle layer from being perforated or damaged by ESD knives by artificially creating an FG clot layer right above the muscle layer, especially for colorectal and duodenal ESD, which has a high perforation rate because of the thin gastrointestinal wall. 
- Table 1 Means and standard deviations, 95\% confidence intervals, and changes for each group.

\begin{tabular}{|c|c|c|c|c|c|c|c|c|c|c|c|c|}
\hline & \multicolumn{4}{|c|}{$\begin{array}{l}\text { Fibrin glue (FG) } \\
(n=5)\end{array}$} & \multicolumn{4}{|c|}{$\begin{array}{l}\text { Hyaluronic acid (HA) } \\
(n=5)\end{array}$} & \multicolumn{4}{|c|}{$\begin{array}{l}\text { Normal saline (NS) } \\
(n=5)\end{array}$} \\
\hline & $\begin{array}{l}\text { Mean } \\
(\mathrm{mm})\end{array}$ & SD & $\begin{array}{l}95 \% \mathrm{Cl} \\
(\mathrm{mm})\end{array}$ & $\begin{array}{l}\text { Amount } \\
\text { of change }{ }^{1} \\
(\mathrm{~mm})\end{array}$ & $\begin{array}{l}\text { Mean } \\
(\mathrm{mm})\end{array}$ & SD & $\begin{array}{l}95 \% \mathrm{Cl} \\
(\mathrm{mm})\end{array}$ & $\begin{array}{l}\text { Amount } \\
\text { of change }{ }^{1} \\
(\mathrm{~mm})\end{array}$ & $\begin{array}{l}\text { Mean } \\
(\mathrm{mm})\end{array}$ & SD & $\begin{array}{l}95 \% \mathrm{Cl} \\
(\mathrm{mm})\end{array}$ & $\begin{array}{l}\text { Amount } \\
\text { of change } 1 \\
(\mathrm{~mm})\end{array}$ \\
\hline $0 \mathrm{~min}$ & 6.2 & 1.1 & $4.9-7.6$ & 0.0 & 6.3 & 0.5 & $5.7-7.0$ & 0.0 & 5.5 & 1.4 & $3.7-7.2$ & 0.0 \\
\hline $5 \mathrm{~min}$ & 5.7 & 0.9 & $4.6-6.8$ & -0.6 & 4.7 & 0.5 & $4.1-5.4$ & -1.6 & 3.1 & 1.0 & $1.8-4.3$ & -2.4 \\
\hline $10 \mathrm{~min}$ & 5.6 & 0.7 & $4.7-6.6$ & -0.6 & 4.0 & 0.6 & $3.3-4.7$ & -2.3 & 2.5 & 1.0 & $1.3-3.8$ & -2.9 \\
\hline $15 \mathrm{~min}$ & 5.6 & 0.7 & $4.7-6.5$ & -0.6 & 3.8 & 0.5 & $3.2-4.4$ & -2.5 & 2.2 & 1.1 & $0.8-3.6$ & -3.3 \\
\hline $20 \mathrm{~min}$ & 5.6 & 0.7 & $4.7-6.5$ & -0.6 & 3.6 & 0.5 & $3.1-4.2$ & -2.7 & 2.0 & 1.1 & $0.6-3.4$ & -3.5 \\
\hline $25 \mathrm{~min}$ & 5.6 & 0.7 & $4.7-6.5$ & -0.6 & 3.4 & 0.4 & $3.0-3.9$ & -2.9 & 1.8 & 1.1 & $0.5-3.1$ & -3.7 \\
\hline $30 \mathrm{~min}$ & 5.6 & 0.7 & $4.7-6.5$ & -0.6 & 3.3 & 0.4 & $2.8-3.8$ & -3.0 & 1.6 & 1.2 & $0.2-3.1$ & -3.9 \\
\hline $45 \mathrm{~min}$ & 5.6 & 0.7 & $4.7-6.5$ & -0.6 & 2.9 & 0.3 & $2.6-3.3$ & -3.4 & 1.6 & 1.2 & $0.1-3.1$ & -3.9 \\
\hline $60 \mathrm{~min}$ & 5.6 & 0.7 & $4.7-6.5$ & -0.6 & 2.7 & 0.2 & $2.5-3.0$ & -3.6 & 1.6 & 1.2 & $0.0-3.1$ & -3.9 \\
\hline
\end{tabular}

${ }^{1}$ Mean change in each sample based on the height of 0 minutes.

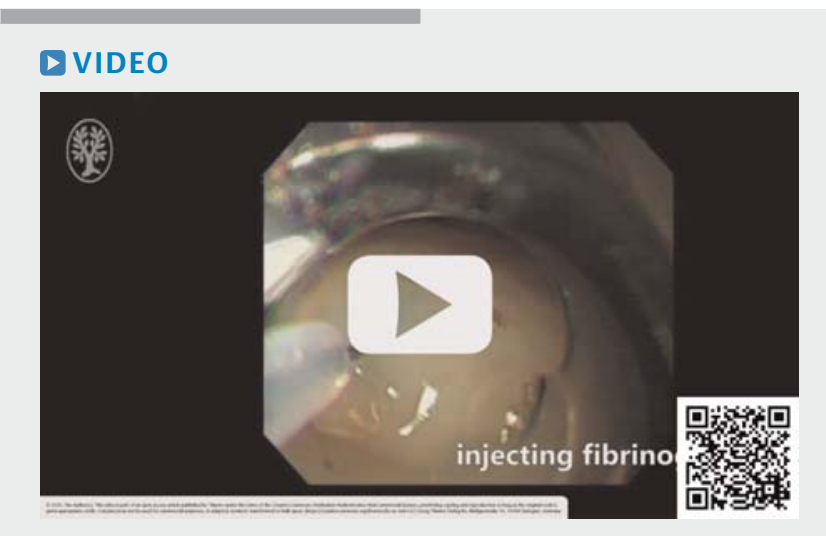

- Video 1 Endoscopic submucosal dissection (ESD) fibrin glue as a submucosal injection agent on the resected porcine stomach. ESD was performed on a hypothetical lesion of approximately $20 \mathrm{~mm}$. The procedure was completed with no technical difficulty.

Our next step is to further examine the availability and effectiveness of FG submucosal injection, including its hemostatic ability and the evaluation of chronological changes in the FG clot condition over time, using live porcine models.

One limitation of the FG solution is its high cost. In the present study, $6 \mathrm{~mL}$ of FG for both solutions was used to resect a 2$\mathrm{cm}$ lesion, which cost 31,383 yen at the Japanese National Health Insurance price. In our clinical experience, one vial of $\mathrm{HA}(20 \mathrm{~mL})$ used for tumor resection of this size costs 7,680 yen, while the cost of NS is only 62 yen $(20 \mathrm{~mL}$ plastic ampoules). The cost of FG solution is approximately four times higher than that of HA. Therefore, application of FG solution should be carefully considered and it should not be routinely used for all ESD cases. Nevertheless, this solution could be use-

\begin{tabular}{|c|c|c|c|}
\hline \multirow[t]{2}{*}{$\begin{array}{l}\text { Lesion } \\
\text { no. }\end{array}$} & $\begin{array}{l}\text { Size of mucosal } \\
\text { defect }(\mathrm{cm})\end{array}$ & $\begin{array}{l}\text { Procedure } \\
\text { time }\end{array}$ & $\begin{array}{l}\text { Total amount of } \\
\text { FG injection }(\mathrm{mL})\end{array}$ \\
\hline & Major $\times$ minor axis & & $\begin{array}{l}\text { Fibrinogen/ } \\
\text { thrombin }\end{array}$ \\
\hline 1 & $2.0 \times 1.8$ & $10 \mathrm{~min} 07 \mathrm{~s}$ & $1.8 / 1.7$ \\
\hline 2 & $2.4 \times 2.0$ & $26 \min 23 s$ & $1.6 / 2.0$ \\
\hline 3 & $2.1 \times 1.9$ & $9 \min 50 \mathrm{~s}$ & $1.9 / 1.9$ \\
\hline
\end{tabular}

ful for patients at high risk for bleeding or perforation, such as in the case of colorectal or duodenal lesions.

Regarding safety, FG has been used in Europe for approximately 20 years as a safe agent of submucosal injection for hemostasis in bleeding ulcer cases, and no report in relevant literature describes major side effects of the FG injection [4-7]. In Japan, FG is not indicated for use as a local injection solution. Potential risks of FG solution are that it is a blood derivative and has the potential to transmit infectious disease. However, after the introduction of nucleic acid amplification test in 1999, FG's safety in relation to infectious disease was established at a high level, and there have been no reported cases of infections caused by this product. Another concern is that when an arterial injection of FG is administered inadvertently, there may be a risk of systemic embolization. However, there have been no reported cases of systemic embolization in patients with endoscopic FG submucosal injection for gastrointestinal ulcer bleeding hemostasis that were confirmed as events caused by FG injection. In an animal model, FG injection has been reported to produce no tissue injury or thrombosis in vessels, suggesting 

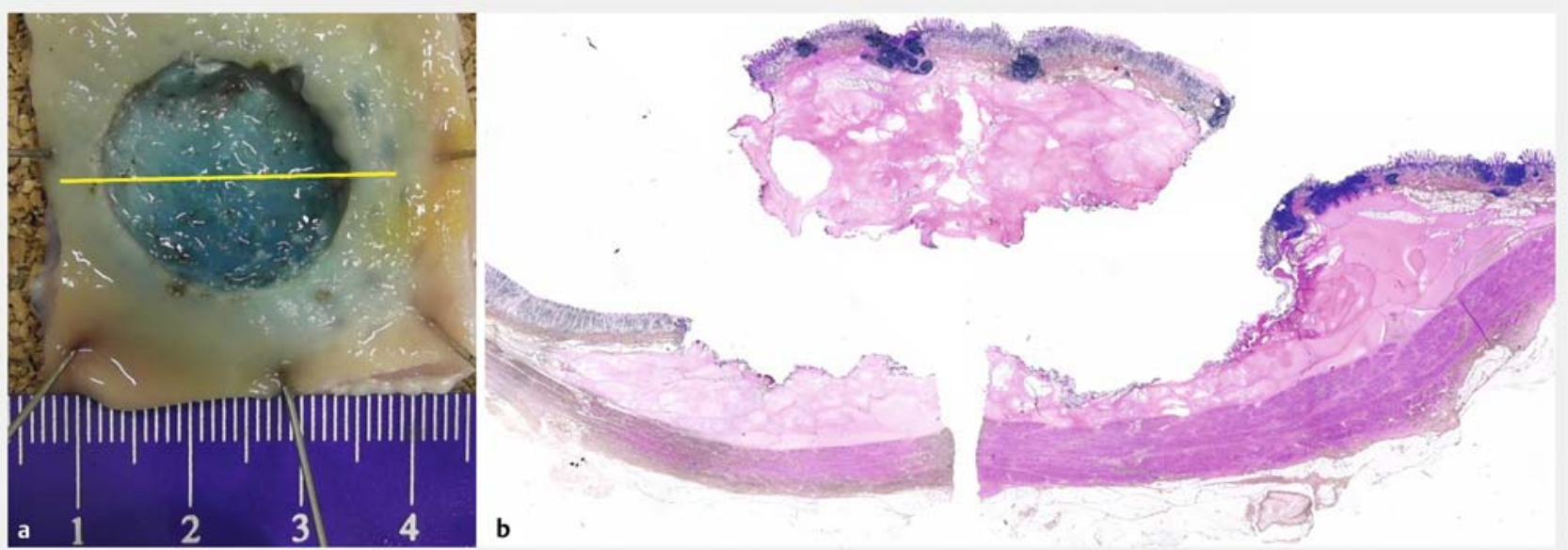

- Fig. 3 Histopathological findings of the ESD ulcer and resected specimen. a Gross image of the ulcer floor. $\mathbf{b}$ Loupe image of a histopathological specimen prepared by splitting the ulcer bed and resected specimen at the yellow line in a. The ulcer is completely covered with FG. Thermal damage or incision to the mucosa was not detected on the resected specimen.

relatively good results, compared with any other examined injection agents [7]. The safety of FG submucosal injection, when used for ESD, should be further assessed in future studies.

\section{Conclusion}

To conclude, FG solution can potentially be used as an ESD submucosal injection agent. However, further investigations using numerous live porcine models must be conducted to confirm the efficacy and safety of the FG injection method.

\section{Acknowledgments}

This research was conducted in collaboration with the Kobe University and KM Biologics Co., Ltd.

\section{Competing interests}

The authors declare that they have no conflict of interest.

\section{References}

[1] Maple JT, Abu Dayyeh BK, Chauhan SS et al. Endoscopic submucosal dissection. Gastrointest Endosc 2015; 81: 1311-1325

[2] Fujishiro M, Yahagi N, Kashimura K et al. Comparison of various submucosal injection solutions for maintaining mucosal elevation during endoscopic mucosal resection. Endoscopy 2004; 36: 579-583

[3] William D. Spotnitz. Fibrin sealant: The only approved hemostat, sealant, and adhesive-a Laboratory and Clinical Perspective. ISRN Surgery 2014: 203943

[4] Rutgeerts P, Rauws E, Wara P et al. Randomised trial of single and repeated fibrin glue compared with injection of polidocanol in treatment of bleeding peptic ulcer. Lancet 1997; 350: 692-696

[5] Pescatore P, Verbeke C, Harle M et al. Fibrin sealing in peptic ulcer bleeding: the fate of the clot. Endoscopy 1998; 30: 519-523

[6] Becker JC, Beckbauer M, Domschke W et al. Fibrin glue, healing of gastric mucosal injury, and expression of growth factors: results from a human in vivo study. Gastrointest Endosc 2005; 61: 560-567

[7] Pundzius ], Jievaltas M. Experimental studies of injection agents for peptic ulcer bleeding endoscopic control. Int Surg 1998; 83: 280-282

[8] Takao T, Takegawa $\mathrm{Y}$, Shinya $\mathrm{N}$ et al. Tissue shielding with polyglycolic acid sheets and fibrin glue on ulcers induced by endoscopic submucosal dissection in a porcine model. Endosc Int Open 2015; 03: E146E151

[9] Oda I, Nonaka S, Abe $S$ et al. Is there a need to shield ulcers after endoscopic submucosal dissection in the gastrointestinal tract? Endosc Int Open 2015; 03: E152-E153

[10] Takao T, Takegawa Y, Ono $\mathrm{H}$ et al. A novel and effective delivery method for polyglycolic acid sheets to post-endoscopic submucosal dissection ulcers. Endoscopy 2017; 49: 359-364 